\title{
Inclusión en deporte adaptado: dos caras de una misma moneda
}

\author{
Raúl Reina \\ Universidad Miguel Hernández de Elche, España
}

(Recibido 20 Abril 2014; Aceptado 27 Abril 2014)

\begin{abstract}
RESUMEN: El presente ensayo pretende mostrar al lector los procesos inclusivos que en la actualidad se están dando en el ámbito del deporte adaptado. En una primera instancia, se aborda el concepto de inclusión, exponiendo las bases filosóficas y prácticas (incluso algunas legislativas) que sustentan la misma, ubicándolo en un modelo actual de consideración del usuario de actividades físicas y deportivas como ente que interactúa con las condiciones de práctica y los elementos que facilitan o dificultan la misma. En un segundo término, se aborda el proceso actual de inclusión para con las organizaciones de deporte adaptado, exponiendo algunas de las controversias derivadas de dicho proceso, especialmente en lo que se refiere a las clasificaciones funcionales, que perpetúan el discurso de las necesidades especiales. Finalizaremos con una propuesta de continuo de inclusión que matiza las posibilidades de práctica inclusiva y específica, pudiendo dar una respuesta más rica y variada a las necesidades del individuo; más allá de las dos caras opuestas de una misma moneda.
\end{abstract}

Palabras clave: deporte paralímpico, discapacidad, integración.

\section{Inclusion in adapted sport: two sides of the same coin}

ABSTRACT: This paper shows current inclusionprocesses in adapted sports. First, some aspects about inclusion are discussed from philosophical and practice perspectives, and its relationship with a functional and health-related model related physical activity and sport activities where client interacts with the personal and environment factors (facilitators and barriers). Second, current inclusion process in sport organizations is explained, exposing some of the disputes arising from this process, especially in regard to functional classifications and its relation with the special needs discourse. Finally, we propose a continuum for inclusion in physical activity and sports, from specific to full inclusion that offers more opportunities and ways of practice; more than two faces of the same coin. Keywords: paralympic sport, disability, integration.

\section{CARA A: ACERCA DE LA INCLUSIÓN}

La Unión Europea se refiere a la inclusión social como un proceso que asegure que personas en riesgo de pobreza y exclusión social aumenten las oportunidades y los recursos necesarios para participar activamente en la vida económica, social y cultural, y de que a su vez, gocen de unas condiciones de vida y de 
bienestar que se consideran normales en la sociedad en la que viven (Ríos et al., 2009). Son muchas las acepciones y matices que adopta la inclusión en actividades físicas y deportes adaptados (sobre este último centraremos nuestra revisión y reflexión), pero podríamos considerar la Inclusión como aquel proceso en el que personas con "discapacidad" comparten mismo espacio, materiales y actividades que sus compañeros (Rouse, 2009). De esta manera, la actividad física inclusiva sería la filosofía y práctica que asegura que todos los individuos, independientemente de su edad y habilidad, tienen iguales oportunidades de práctica en actividad física (Kasser, y Little, 2005).

El Artículo 31 de la Convención de la Organización de Naciones Unidas sobre los derechos de las personas con discapacidad (Naciones Unidas, 2006) establece que los adultos y niños con discapacidad deben tener un acceso a actividades de ocio, recreación y deporte, tanto en entornos inclusivos como específicos. Esta disposición legislativa internacional se materializa en nuestro país, amén de otras muchas acciones de buenas prácticas (Pérez-Tejero, 2013; Comité Paralímpico Español, 2014), en el BOE de 21 de abril de 2008, que ratifica el citado artículo de las Naciones Unidas sobre participación en la vida cultural, las actividades recreativas, el esparcimiento y el deporte. Algunas de las recomendaciones propuestas para conseguir dicho objetivo serían:

a) alentar y promover la participación, en la mayor medida de lo posible, de las personas con discapacidad en las actividades deportivas generales a todos los niveles, b) asegurar que las personas con discapacidad tengan la oportunidad de organizar y desarrollar actividades deportivas y recreativas especificas para dichas personas; c) asegurar que las personas con discapacidad tengan acceso a instalaciones deportivas, recreativas y turisticas; d) asegurar que los niños y niñas con discapacidad tengan igual acceso que los demás niños y niñas a la participación en actividades lúdicas, recreativas, de esparcimiento y deportivas, incluidas las que se realicen dentro del sistema escolar; y e) asegurar que las personas con discapacidad tengan acceso a los servicios de quienes participan en la organización de actividades recreativas, turísticas, de esparcimiento $y$ deportivas.

La International Classification of Functioning, Disability and Health -CIF(O.M.S., 2001) permitió transformar una clasificación basada en el déficit a una clasificación basada en competencias, de componentes de salud. El término de funcionalidad (functioning) se refiere a todas las funciones corporales, actividades y de participación, mientras que el de discapacidad (disability) sería su "antítesis" en cuanto a impedimentos, limitación en las actividades y restricciones en la participación. Ello supone un cambio relevante sobre cómo se entiende actualmente la "discapacidad", cambiando el foco de atención al estado de salud y capacidades del usuario (Reina, 2014). Además, la CIF provee el uso de un lenguaje estandarizado y un marco teórico para la descripción de diferentes estados relacionados con la salud, y su aplicación en la actualidad va más allá del ámbito sanitario. Algunos modelos de actividad física inclusiva se basan en los elementos de la CIF, donde una serie de factores condicionarían el desarrollo de 
dicho progreso inclusivo, tales como (Kasser, y Lytle, 2005): a) factores personales (conocimiento, motivación, riesgos percibidos...), b) factores contextuales (actitudes, lenguaje, competencia profesional, accesibilidad, apoyo administrativo...), y c) factores de las tareas/actividades (equipamientos, actividades propuestas). Así, el éxito del proceso inclusivo radica en su esencia en las interacciones que se dan entre la persona y las tareas planteadas, lo que implica un ajuste en función de las capacidades de la persona, usuario, deportista o estudiante.

Según algunos autores que han abordado los procesos inclusivos en educación física (Block, 2000; Block, y Obrusnikova, 2007), así como otros contextos de actividad física y deporte (De Pauw, y Doll-Tepper, 2000;Pérez-Tejero, 2013), encontramos una serie de postulados relacionados con el usuario o participante en actividades inclusivas, tales como que cada persona es única, con diferentes capacidades y necesidades físicas, cognitivas, emocionales y sociales; que cada persona tiene el derecho a beneficiarse de actividades físicas inclusivas; que las habilidades de una persona varían y son el resultado de la relación entre el individuo, el contexto de prácticas y las tareas/actividades realizadas; que los usuarios tienen derecho a elegir y tomar sus propias decisiones; o que cada individuo puede beneficiarse de la experiencia de los demás. Así, los beneficios de las prácticas inclusivas han sido ampliamente reportados para sus practicantes, entre los que podemos destacar el respeto por las diferencias y habilidades individuales, un conocimiento más profundo de las fortalezas y debilidades propias (Lindsay, McPherson, Aslam, McKeever, y Wright, 2013), un mayor espectro de oportunidades y experiencias (Block, Taliaferro, Campbell, Harris, y Tipton, 2011), experiencias en un entorno con más motivación y normalización (PérezTejero,Ocete, Ortega-Vila, y Coterón, 2012), desarrollo de recursos para evitar el aislamiento con respecto a otros significativos, incremento del sentimiento de aceptación y comunidad (Suomi, Collier, y Brown, 2003), incremento de contribuir a los objetivos y resultados de los programas, o el aumento del valor individual y autoestima (Martin, y Smith, 2002).

Partimos de la premisa de que el modelo ICF se presenta como un marco de referencia transversal, que permitiría abordar el concepto de salud desde perspectivas individuales, biológicas y sociales. La interacción entre la persona con diversidad funcional y el entorno de práctica hace que los propios programas deportivos, así como el resto de usuarios y responsables de los mismos, se nutran de postulados como (Kasser, y Little, 2005; Reina, Martínez-Galindo, CebriánSánchez, e Iñiguez-Santiago, 2014): el valor de la diversidad de los usuarios que participan en los programas, la consideración de los intereses y necesidades individuales para una práctica equitativa, el ofrecimiento de actividades físicas y deportivas significativas y motivantes, o el respeto por las necesidades de práctica de usuarios con diversidad funcional. La consecuencia de ello sería pues también una serie de beneficios, entre los que podríamos destacar la sensibilización y conocimiento del colectivo (Reina, López, Jiménez, García-Calvo, y Hutzler, 2011), un cambio de perspectiva sobre la práctica profesional (Leo, y Goodwin, 2014), aumento de los recursos y estrategias para la práctica, au- 
mento del conocimiento sobre variantes de las tareas y habilidades demandadas (Block et al., 2011), o conocer el valor de trabajar con personas con diferentes habilidades(Lieberman, y Houston-Wilson, 2009).

Hasta este punto hemos expuesto las bondades del proceso inclusivo, pero atendiendo al título de este ensayo, no podemos olvidar otra cara de este proceso: aquellas barreras y limitaciones que apuntamos sucintamente al hablar de "discapacidad" cuando expusimos los postulados de la CIF. Las barreras, así como su alter-ego los elementos facilitadores, podríamos dividirlos en factores personales y del entorno. Una reciente revisión sistemática realizada por Jaarsma, Dijkstra, Geertzen, y Dekker (2014) concluye que las principales barreras personales radican en la propia discapacidad, la salud o la falta de energía/fatiga a la hora de realizar las actividades deportivas. Sin embargo, una adecuada percepción del estado de salud hace que éste se convierta en un elemento facilitador, en la medida que impulsaría la participación en programas deportivos. Las limitaciones del entorno más destacadas fueron la falta de oportunidades de práctica deportiva, las dificultades de accesibilidad y transporte a las mismas, la falta de información sobre la oferta existente, o los costes derivados para las prácticas, especialmente en lo que se refiere al acceso a material deportivo específico.

El informe realizado por Ríos et al. (2009) muestra además una serie de condicionantes sociales como el desconocimiento de la población general de las necesidades del colectivo con diversidad funcional, que puede derivar en situaciones de evitación, insolidaridad o falta de sensibilización; el escaso conocimiento de la rentabilidad social que implica el dotar de igualdad de oportunidades o programas adecuados a las necesidades de los usuarios; la formación insuficiente (o precaria) de algunos profesionales de la actividad física y del deporte; las ideas preconcebidas de oferta de programas específicos ("segregados"), que pueden limitar el avance hacia un enfoque inclusivo; la actitud de las propias familias de las personas con diversidad funcional, ya sea por desconocimiento de los beneficios, miedos injustificados hacia la práctica deportiva (sobreprotección) o la falta de tiempo para poder dar el soporte necesario; así como un deficiente trabajo en red y falta de coordinación institucional.

\section{CARA B: APLICACIÓN AL DEPORTE ADAPTADO}

Extrapolando el movimiento inclusivo al ámbito del deporte, partiremos de una concepción de Deporte Adaptado que englobaría a todas aquellas modalidades deportivas que se adecúan a personas con diferentes capacidades, bien porque se han realizado una serie de adaptaciones y/o modificaciones para facilitar la práctica a ese colectivo, o porque la propia estructura del deporte permite su práctica sin adaptaciones relevantes (Sanz, y Reina, 2010). Pero el deporte adaptado tiene muchas acepciones, que van desde el deporte salud, para todos o recreativo, donde el proceso inclusivo se torna más factible; al deporte de competición o de alto rendimiento (Sanz, y Reina, 2012). Dado que en el primer apartado de este ensayo hemos hablado de las bondades (y barreras) de la actividad 
deportiva inclusiva, nos centraremos a continuación en el deporte federado y/o de competición.

Cual avance más hacia la inclusión en diferentes esferas sociales, numerosas Federaciones Deportivas Internacionales han dado instrucciones a sus respectivas Federaciones Nacionales para que acojan la modalidad de deporte adaptado dentro de sus estructuras organizativas y de práctica deportiva. Este proceso ha permitido responder a uno de los objetivos reivindicados por las personas con discapacidad: la integración e inclusión en la sociedad y en todas sus estructuras y ámbitos sociales (Jofre, 2009). En palabras del propio Alberto Jofre, Director Gerente del Comité Paralímpico Español, la integración en las federaciones deportivas lograría una mayor especialización de los servicios ofrecidos a los deportistas con discapacidad y a sus técnicos para la práctica de su deporte. Además, la integración produciría beneficios como el incremento del número de practicantes de actividad física y deportiva entre las personas con discapacidad; elevar las cuotas de cohesión del tejido social y favorecer la inclusión del colectivo de personas con discapacidad; rentabilizar los recursos destinados a la promoción del deporte y de la actividad física; y sensibilizar a todos los agentes involucrados en la promoción del deporte y de la actividad física sobre la igualdad de oportunidades.

Este proceso está muy activo en estos momentos. Si tomamos como referencia los deportes incluidos en los programas de verano e invierno de los Juegos Paralímpicos $(n=26)$, estamos en un punto de inflexión en el que ya algo más de la mitad de los deportes desarrollan su actividad al amparo de una federación deportiva específica, ya sea de un deporte ordinario con su sección de deporte adaptado o paralímpico $(\mathrm{n}=9)$ (e.g. ciclismo está como una sección de paraciclismo en la Union Cycliste Internationale -UCI-); o como entidad representativa del propio deporte adaptado $(n=6)$ (e.g. baloncesto en silla de ruedas está gobernadopor la International Wheelchair Basketball Federation -IWBF-). Por otro lado, tendríamos aquellas entidades representativas de diferentes colectivos específicos de discapacidad (conocidas como International Organizations of Sports for the Disabled -IOSD-), o bien específicas de un deporte paralímpico en concreto. Así, encontramos que IWAS (International Wheelchair and Amputee Sports Federation) sólo acoge en la actualidad la esgrima;Cerebral Palsy International Sports and Recreation Association (CPISRA) sólo acoge fútbol-7 (que será independiente de dicha federación en enero de 2015), y la International Blind Sports Federation (IBSA), que acoge tres deportes para el colectivo de ceguera y deficiencia visual (judo, goalball, y fútbol 5). En última instancia, el propio International Paralympic Committee(IPC) acogería el gobierno de 8 deportes paralímpicos que, por diferentes motivos, no han podido (todavía) integrase en las estructuras de ese deporte en cuestión (e.g. atletismo o natación). A ello habría que sumar el movimiento deportivo para personas con discapacidad auditiva (International Committee of Sports for the Deaf, con 24 deportes en su programa Deaflympics), o el de Special Olympics para personas con discapacidad intelectual $(\mathrm{n}=32)$. 
Pero el deporte paralímpico, tal y como lo conocemos en nuestros días, es entendido como élite, inspiración y excitación (Tweedy, y Howe, 2011), donde el deportista puede desarrollar un empoderamiento personal que tenga impacto en su salud, bienestar y autoestima (Wedgood, 2014a). Cualquier deporte surge de un contexto social o histórico concreto (Anderson, 2003), pero en el caso del deporte paralímpico éste lo hizo en un contexto médico, complementario a la rehabilitación física, social y psicológica, concretamente de personas con paraplejias (Gutmann, 1976; Sanz, y Reina, 2012). Howe (2008) hace una clasificación acerca del desarrollo histórico del deporte paralímpico en tres fases: a) una primera fase en ese contexto de rehabilitación que acabamos de comentar, b) un movimiento que proporciona oportunidades de participación a todas aquellas personas con discapacidad física para competir con otros iguales; y c) la situación actual de deporte de élite, considerado un espectáculo, y sujeto a los intereses comerciales del deporte contemporáneo. Así, hemos asistido en la última década a un proceso de colaboración entre los comités internacionales olímpicos y paralímpicos (Doll-Tepper, 1999), con la desventaja para estos últimos de que en ocasiones los procesos de toma de decisiones recaen en representantes alejados de la realidad de las personas con diversidad funcional (Howe, 2008). Paradójicamente, esta situación se contrapone a los postulados del movimiento inclusivo que expusimos al principio de este ensayo, donde la persona con diversidad funcional debe ser una parte activa del programa en el que participa.

Llegados a este punto, cabe indicar que, en el contexto del deporte federado o de competición, los procesos de inclusión se tornan más complejos, al entrar en escena las clasificaciones funcionales, que progresivamente aplican a su vez los postulados de la CIF (Tweedy, y Vanlandewijck, 2011). Alguno de los objetivos de tales clasificaciones deportivas, inmersas actualmente en un importante proceso de revisión y giro hacia sistemas de clasificación basados en evidencias, son: minimizar el impacto del impedimento del deportista en el resultado de la competición; que el éxito en la competición dependa de factores de entrenamiento, destreza o capacidades innatas; que la clasificación evidencie el impacto que el impedimento tiene sobre la actividad de cada deporte; estimular la participación en deporte competitivo; o prevenir el abandono deportivo de deportistas con severos impedimentos o limitaciones funcionales.

Howe (2008) considera que estos sistemas de clasificación son una forma de gobierno del deporte paralímpico, en la medida que están desarrollados generalmente por personas sin discapacidad y puede llevar a la estigmatización, generando una jerarquía de cuerpos (Brittain, 2010). Dado que los primeros sistemas de clasificación se basaron en el tipo y grado de discapacidad para crear una práctica competitiva lo más equitativa posible, también es cierto que el número de eventos era demasiado grande, lo que dificultaba la logística de los eventos y hacía que el deporte no pudiera ser tan atractivo para su esponsorización. La consecuencia de todo ello ha sido la supresión de determinadas clases funcionales de los grandes eventos deportivos, aquellos que al fin y al cabo son los que llegan al gran público, con especial impacto en las clases de deportistas con mayor li- 
mitación funcional. Otra de las consecuencias sería a veces la eliminación de los programas deportivos de máximo nivel de pruebas específicas que pudieran ser consideradas (por lo gestores del deporte paralímpico) "poco atractivas" para el público general.

Ante esta situación, nos podríamos hacer la pregunta de si el deporte adaptado es en este contexto inclusivo o exclusivo. El propio Gutmann, padre del movimiento paralímpico, argumentaba que la competición de gente con y sin discapacidad "permitiría mejorar la comprensión entre las personas con y sin discapacidad, y ayudar a los últimos a su reintegración social" (Guttmann, 1976: p. 13). Nuevamente, nos encontramos con la paradoja de que el deporte adaptado de rendimiento (paralímpico) ha estado separado o, desde los juegos olímpicos y paralímpicos de Seúl en 1988, ha sido paralelo a unos juegos olímpicos, por lo que podría perpetuarse el discurso de las "necesidades especiales" (Goggin, y Newell, 2000).Tradicionalmente ha existido el debate acerca de si esta inclusión del deporte paralímpico en relación con el olímpico es lo mejor para los primeros (Labanovich, 1988;Steadward, 1996; Von Selzam, 2001), hasta el punto en que se podría cuestionar si los juegos paralímpicos pueden ser contraproducentes para los derechos de las personas con discapacidad, ya que una gran parte de la población con discapacidad no estaría representada (Braye, Dixon, y Gibbons, 2013). No obstante, si se avanzara en la inclusión de deportistas con discapacidad en eventos competitivos normalizados, la diferenciación binaria que acabamos de hacer sería cada vez más borrosa (Wedgwood, 2014b), por lo que no podemos asumir que esta integración expuesta a nivel deportivo (de élite u otro nivel inferior) automáticamente represente lo que ocurre a nivel social.

\section{UN CONTINUO PARA LA INCLUSIÓN EN DEPORTE ADAPTADO}

De una forma simple y concreta, el Plan Integral para la Actividad Física y el Deporte, en su apartado de Personas con Discapacidad (Ríos et al, 2009), plantea dos tipos de práctica deportiva: a) práctica del deporte y de la actividad física en grupo inclusivo, referida a la persona con discapacidad que comparte todas las actividades físico deportivas con personas sin discapacidad; y b) práctica de deporte y actividad física en grupo específico, referida a la práctica de personas con discapacidad, realizando un trabajo conjunto, al margen del ordinario.

Al margen de los ámbitos de aplicación de los programas deportivos para personas con diversidad funcional (Sanz, y Reina, 2010; 2012), es interesante dirigir nuestra atención acerca de la forma de llevarlos a cabo, en un continuo que vaya desde situaciones de reducida a máxima inclusión -Figura 1- (propuesta modificada de Paciorek, 2011). El nivel 1 representaría el programa de deporte adaptado específico (al que Paciorek denomina segregado), donde el usuario con discapacidad realizaría la actividad deportiva al margen de un grupo de persona sin discapacidad. Este tipo de programas podrían estar justificados en cuanto a los requerimientos de práctica o que los materiales sean específicos y exclusivos del colectivo (e.g. empleo de canaletas para la práctica de boccia). La prevalen- 
cia de estos programas perdura en nuestro días por la existencia de las entidades organizativas representativas de diferentes grupos de discapacidad (discapacidad física, parálisis cerebral y daño cerebral, discapacidad visual, discapacidad auditiva, y discapacidad intelectual) (Reina, Menayo, y Sanz, 2011).Un paso más adelante, el nivel 2 lo podríamos denominar deporte adaptado integrado, donde personas sin discapacidad podrían tomar parte de los programas de práctica deportiva de un colectivo o deporte adaptado. Este nivel de práctica permite que la persona sin discapacidad participe en el programa en la misma situación que lo haría una persona con discapacidad, adecuándose así a un entorno de práctica común. Este tipo de acciones son de gran interés cuando tenemos dificultad de potenciar o desarrollar programas específicos, además de disponer de una herramienta de sensibilización de primer orden (Reina et al., 2011). Algunos ejemplos los podemos encontrar en una persona que se siente en una silla de ruedas para jugar al baloncesto, se ponga un antifaz para jugar a goalball, o use unas muletas para jugar al fútbol para personas con amputación. Esta forma de integración ha sido también denominada por algunos autores como integración inversa (Brasile, 1992; Sørensen, y Kahrs, 2006).

El punto de inflexión de esta propuesta adaptada se ubicaría en el nivel 3, donde se encuentran las realidades del deporte adaptado y el deporte regular $\mathrm{u}$ ordinario, en entornos que son parcialmente o totalmente inclusivos. Un ejemplo lo podríamos encontrar en un partido de tenis de dobles, en el que un miembro de una pareja va en silla de ruedas y el otro no, de manera que se permite el doble bote de la bola para el deportista en silla de ruedas. Otro ejemplo a este nivel sería el programa Deportes Unificados de Special Olympics, donde deportistas con discapacidad intelectual y sin discapacidad participan conjuntamente (e.g. en un equipo de baloncesto habrían 3 jugadores con discapacidad intelectual y 2 compañeros). En este contexto, los usuarios realizan la práctica deportiva de acuerdo a un objetivo común, con normativas y condicionantes de práctica que pueden adecuarse a las necesidades de cada usuario.

El nivel 4 representa, esencialmente, entornos regulares de práctica deportiva, distinguidos únicamente por la necesidad de acomodación, por lo que se permite que el deportista con discapacidad participe en programas de deporte normalizado, empleando para ello alguna acomodación (p.e. uso de bandas laterales en una pista de bolos para personas con ceguera, o el empleo de un dispositivo de lanzamiento para una persona con lesión medular). Esta acomodación (elemento facilitador) no debería suponer una desventaja para la persona con discapacidad, y debe proporcionarle las mismas oportunidades para obtener los beneficios o resultados que los otros participantes. Finalmente, el nivel 5 representaría la situación de mayor normalización, donde los deportistas con discapacidad participan conjuntamente en un contexto deportivo particular. Uno de los ejemplos mediáticos en este sentido, y de gran controversia en los últimos años, ha sido la participación de Oscar Pistorius en los pasados Juegos Olímpicos (y Paralímpicos) de Londres 2012, donde se cuestionaba si las prótesis utilizadas aportaban alguna ventaja en el rendimiento deportivo (Weyand, Bundle, 
McGowan, Grabowski, Brown, Kram, y Herr, 2009). Pero este debate perdura en la actualidad, y puede incluso extenderse a otros deportistas que requieran de ayudas técnicas para la posibilidad de esa práctica inclusiva de rendimiento. Un nuevo caso en el futuro próximo podría darse con el atleta brasileño Alan Oliveira, quien batió a Pistorious en la final de 200m de los pasados Juegos Paralímpicos de Londres. Si Oliveira decidiera participar en un evento inclusivo con deportistas sin discapacidad, éste deberá nuevamente someterse a un estudio acerca del uso de sus prótesis, ya que no existe una reglamentación vigente que regule el uso de este material técnico adaptado (Wolpe, 2012), debido a que las directrices desarrolladas en el caso Pistorius para el uso de las prótesis fueron especificas de ese caso en particular.

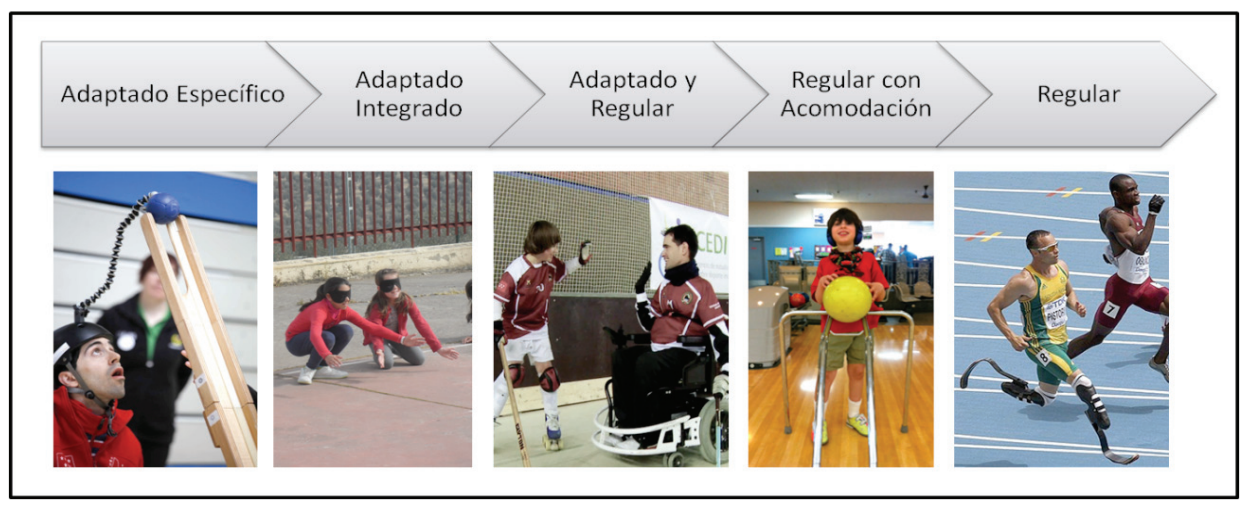

Figura 1. Contínuo de práctica deportiva hacia la plena inclusión.

En cualquiera de los niveles o formas que se practique el deporte adaptado, éste se hace preciso para el colectivo con discapacidadpara evitar ciertos problemas de salud (Rimmer, Schiller, y Chen, 2012), por lo que existe cierta urgencia para su promoción y de la actividad física en general (Rimmer, y Marques, 2012). Si bien al principio de este ensayo hablamos de las barreras que dificultan dicha práctica (la cara menos amable de nuestra moneda), debemos actuar sobre aquellos elementos facilitadores que ayudarían a su promoción. Desde el prisma personal, la diversión aparece como uno de los elementos favorecedores de la participación en programas deportivos adaptados (e.g. Saebu, y Sorensen, 2011), así como la mejora de salud (Dlugonski, Wojcicki, McAuley, y Motl, 2011) y el estado de forma ofitness (Beckerman, de Groot, Scholten, Kempen, y Lankhorst, 2010). Desde un punto de vista más psicosocial, cabe indicar que algunos elementos facilitadores reportados en la literatura son la motivación intrínseca del usuario (Saebu, y Sorensen, 2011), la autonomía (Plow, Finalayson, y Cho, 2011) o la búsqueda de relaciones sociales (Kars, Hofman, Geertzen, Pepping, y Dekker, 2009). 


\section{CONCLUSIONES}

Hemos pretendido con el presente ensayo mostrar al lector la realidad actual entorno a los procesos de inclusión en deporte adaptado, y plantear una propuesta de continuo de inclusión que matice las posibilidades de práctica inclusiva y específica. La participación en actividades físicas y/o deportivas está condicionada por una serie de factores personales y del contexto, que pueden hacer de agentes facilitadores o barreras. Metafóricamente, y haciendo referencia al título de este trabajo, unos y otros hacen que se muestre una u otra cara de esta moneda. Además, el deporte de competición, aquel que llega más al público general o tiene más cobertura (poca, por cierto) en los medios de comunicación, presenta la realidad de las clasificaciones funcionales, que pueden perpetuar la categorización de los deportistas con diversidad funcional, creando una contradicción con la igualdad de derechos alcanzados en otras facetas.

\section{AGRADECIMIENTOS}

El autor agradece la invitación para la publicación de este ensayo en la revista Psychology, Society, \& Education tras la ponencia "Inclusión en deporte adaptado: estrellas y agujeros negros" del II Congreso Nacional de Sensibilización al Estigma en Salud Mental y III Internacional de Actividad Física y Deporte Adaptado (Almería, 9 a 12 de abril de 2014)

\section{REFERENCIAS}

Anderson, J. (2003). Turned into taxpayers: Paraplegia, rehabilitation and sport at Stoke Mandeville, 1944-56. Journal of Contemporary History, 38(3), 461475.

Beckerman, H., de Groot, V., Scholten, M. A., Kempen, J., y Lankhorst, G. J. (2010). Physical activity behavior of people with multiple sclerosis: understanding how they can become more physically active. Physical Therapy, 90, 1001-1013.

Block, M. E. (2000). A teacher's guide to including students with disabilities in regular physical education (2nd ed.). Baltimore: Paul H. Brookes.

Block, M. E., y Obrusnikova, I. (2007). Inclusion in physical education: a review of the literature from 1995-2005. Adapted Physical Activity Quarterly, 24, $103-124$.

Block, M. E., Taliaferro, A.; Campbell, A. L., Harris, N., y Tipton, J. (2011).Teaching the self-contained adapted physical education class.Journal of Physical Education, Recreation and Dance, 82 (4), 47-52.

Brasile, F. (1992). Inclusion: A developmental perspective. A rejoinder to "Examining the concept of Reverse Integration".Adapted Physical Activity Quarterly, 9, 293-304.

Braye, S., Dixon, K., y Gibbons, T. (2013). “A mockery of equality”: An explor- 
atory investigation into disabled activists' views of the Paralympic Games. Disability and Society, 28(7), 984-996.

Brittain, I. (2010). The paralympic games explained. Oxon: Routledge.

Comité Paralímpico Español (2014). La inclusión en actividad física y deportiva. Barcelona: Paidotribo.

De Pauw, K. P., y Doll-Tepper, G. (2000). Toward progressive inclusion and acceptance: Myth or reality? The inclusion debate and bandwagon discourse. Adapted Physical Activity Quarterly, 17, 135-143.

Doll-Tepper, G. (1999). Disabiity sport. London: E \& F N Spon.

Dlugonski, D., Wojcicki, T. R., McAuley, E., y Motl, R. W. (2011). Social cognitive correlates of physical activity in inactive adults with multiple sclerosis. International Journal of Rehabilitation Research, 34, 115-120.

Goggin, G., y Newell, C. (2000). Crippling Paralympics? Media, disability and olympism.Media International Australia incorporating Culture and Policy, 97, 71-83.

Guttmann, L. (1976). Textbook of sport for the disabled. Brisbane: University of Queensland Press.

Howe, P. D. (2008). The cultural politics of the paralympic movement: Through an anthropological lens. London: Routledge.

Jaarsma, E. A., Dijkstra, P. U., Geertzen, J. H., y Dekker, R. (2014). Barriers to and facilitators of sports participation for people with physical disabilities: A systematic review. doi: 10.1111/sms.12218. [Epub ahead of print]

Jofre, A. (2009). El reto de la integración del deporte adaptado español en el deporte convencional. En J. Pérez, y D. Sanz (Eds.), Libro de Actas de la I Conferencia Nacional de Deporte Adaptado (CD-Rom, Bloque I). Toledo, Consejo Superior de Deportes.

Kars, C., Hofman, M., Geertzen, J. H., Pepping, G. J., y Dekker, R. (2009). Participation in sports by lower limb amputees in the Province of Drenthe, the Netherlands.Prosthetics and Orthotics International, 33, 356-367.

Kasser, S. L., y Lytle, R. K. (2005). Inclusive physical activity.A lifetime of opportunities. Champaign, IL: Human Kinetics

Labanowich, S. (1988).A case for the integration of the disabled into the Olympic Games.Adapted Physical Activity Quarterly, 5, 264-272.

Leo J., y Goodwin, D. (2014). Negotiated meanings of disability simulations in an adapted physical activity course: Learning from student reflections. Adapted Physical Activity Quarterly, 31, 144-161.

Lieberman, L. J., y Houston-Wilson, C. (2009). Strategies for inclusion (2nd ed.). Champaign, IL: Human Kinetics.

Lindsay, S., McPherson, A. C., Aslam, H., McKeever, P., y Wright, V. (2013). Exploring children's perceptions of two school-based social inclusion programs: A pilot study. Child and Youth Care Forum, 42 (1), 1-18.

Martin, J. J., y Smith, K. (2002). Friendship quality in youth disability sport: Perceptions of a best friend. Adapted Physical Activity Quarterly, 19(4), 472482. 
NacionesUnidas (2006). UN Convention on the rights of persons with disabilities and optional protocol 2006. New York, NY: United Nations.

O.M.S. (2001). International Classification of Functioning Disability and Health.Extraído el 25 de febrero de 2014 desde http://www.who.int/classifications/icf/en/

Paciorek, M. J. (2011). Adapted sport. En J. P. Winnick (Ed.), Adapted physical education and sport (pp. 41-58). Champaign, IL: Human Kinetics.

Pérez-Tejero, J. (2013). Centro de estudios sobre deporte inclusivo. Cuatro años de fomento del deporte inclusivo a nivel práctico, académico y científico. Madrid: Universidad Politécnica de Madrid, Sanitas y Psysport.

Pérez-Tejero, J., Ocete, C., Ortega-Vila, G., y Coterón, J. (2012). Diseño y aplicación de un programa de intervención de práctica deportiva inclusiva y su efecto sobre la actitud hacia la discapacidad: El Campus Inclusivo de Baloncesto. Revista Internacional de Ciencias del Deporte, 29, 258-271.

Plow, M. A., Finlayson, M., y Cho, C. (2011). Correlates of stages of change for physical activity in adults with multiple sclerosis. Research in Nursing and Health, 34, 378-388.

Reina, R., López, V., Jiménez, M., García-Calvo, T., y Hutzler, Y. (2011). Effects of awareness interventions on children's attitudes toward peers with a visual impairment.International Journal of Rehabilitation Research, 34(3), 235-242.

Reina, R., Menayo, R., y Sanz, D. (2011). Cómo se organiza el deporte adaptado a las personas con discapacidad física. En J. Palau, J. O. Martínez, M. A. García, y M. Ríos (Eds.), Deportistas sin adjetivos. El deporte adaptado a las personas con discapacidad física (pp. 117-132). Madrid: Cromagraf.

Reina, R. (2014). Adapted Physical Activity: the journey to Ithaca goes ahead. International Journal of Sport Science, 37, 177-179.

Reina, R., Martínez-Galindo, M. C., Cebrián-Sánchez, M., e Iñiguez-Santiago, M. C. (2014). Efecto de 4 intervenciones de sensibilización hacia la inclusión de alumnos con discapacidad en Educación Física. Comunicación presentada en el 1er Simposium Internacional de Primavera en Enseñanza FísicoDeportiva fundamentada en Evidencias, Elche, España.

Rimmer, J. H., y Marques, A. C. (2012). Physical activity for people with disabilities. The Lancet, 380, 194-195.

Rimmer, J. H., Schiller, W., y Chen, M. D. (2012).Effects of disability-associated low energy expenditure deconditioning syndrome. Exercise and Sport ScienceReviews, 40, 22-29.

Ríos, M. et al. (2009). Plan integral para la actividad Física y el Deporte: Personas con Discapacidad. Extraído el 28 de mayo de 2014 desde http://www.csd. gob.es/csd/estaticos/plan-integral/discapacidad.pdf.

Rouse, P. (2009). Inclusion in physical education. Champaign, IL: Human Kinetics

Saebu, M., y Sorensen, M. (2011).Factors associated with physical activity among young adults with a disability. Scandinavian Journal of Medicine and 
Science in Sports, 21, 730-738.

Sanz, D., y Reina, R. (2010). Informe experto APRADDIS: Ámbito deportivo. En J. Pérez (Coord.), Análisis de la práctica de actividad físico deportiva de personas con discapacidad en España: Hábitos deportivos, estudio demográfico y medidas de promoción (APRADDIS). Madrid: Centro de Estudios del Deporte Inclusivo.

Sanz, D., y Reina, R. (2012). Actividades físicas y deportes adaptados para personas con discapacidad. Barcelona: Paidotribo.

Sørensen, M., y Kahrs, N. (2006). Integration of disability sport in the Norwegian sport organizations: Lessons learned. Adapted Physical Activity Quarterly, 23(2), 184-203.

Steadward, R. (1996). Integration and sport in the paralympic movement. Sport Science Review, 5(1), 26-41.

Suomi, J., Collier D., y Brown, L. (2003).Factors affecting the social experiences of students in elementary physical education classes. Journal of Teaching in Physical Education, 22(2), 186-202.

Tweedy, S., y Howe, P. D. (2011).Introduction to the paralympic movement. En Y. C. Valandewijck, y W. R. Thompson (Eds.), Theparalympic athlete (pp. 3-32). Chichester, WS: Wiley-Blackwell.

Tweedy, S., y Vanlandewijck, Y. (2011).International Paralympic Committee position stand - background and scientific principles of classification in $\mathrm{Pa}$ ralympic sport.British Journal of Sports Medicine, 45, 259-269.

vonSelzam, H. (2001). Paralympic and Olympic Games - Separate or together?. En G. Doll-Tepper, M. Kröner, y W. Sonnenschein (Eds.), New horizons in sport for athletes with a disability (pp. 583-597). Oxford: Meyer \& Meyer Sport.

Wedgwood, N. (2014a). My biggest disability is I'm a male! The role of sport in negotiating the dilemma of disabled masculinity. En J. Hargreaves, y E. Andersen (Eds.), Routledge handbook of sport, gender and sexuality (pp. 189197). London: Routledge.

Wedgwood, N. (2014b). Hahn versus Guttmann: revisiting "Sports and the political movement of disabled persons". Disability and Society, 29(1), 129-142.

Weyand, P. G., Bundle, M. W., McGowan, C. P., Grabowski, A., Brown, M. B., Kram, R., y Herr, H. (2009). The fastest runner on artificial legs: different limbs, similar function? Journal of Applied Physiology, 107, 903-911.

Wolpe, P. (2012). Oscar Pistorius, an inspiration and a question. CNN, $7 \mathrm{Au}-$ gust 2012. Extraído el 14 de mayo de 2014 desde http://edition.cnn. com/2012/08/07/opinion/wolpe-oscar-pistorius/ 